Research Journal of Agronomy 4 (4): 70-77, 2010

ISSN: $1815-9354$

(C) Medwell Journals, 2010

\title{
Effects of Light-Temperature Factors on the Grain Yield and Yield Components in Different Latitude Areas
}

\author{
Liu Wen-Jiang and Yang Wen-Yu \\ Key Laboratory of Crop Culture and Tillage Science of Sichuan Agricultural University, \\ Sichuan, 625014 Ya'an, P.R. China
}

\begin{abstract}
For a better understanding of the effects of light-temperature factors on the grain yield and yield components in different latitude areas, we have done six field experiments in Shanxi Hanzhong, Sichuan Wenjiang, Yunnan Mile of China and Hanoi Wenlin, Zhongbu Guang'ai, Saigon Long'an of Vietnam from 2005-2006. The results showed as follows: there was real linear regression between seven light-temperature factors and grain yield. The size order of standard regression coefficient was: cumulateive illumination intensity $>$ duration of day $>$ average temperature $>$ average illumination intensity $>$ maximum temperature $>$ effective accumulated temperature $>$ minimum temperature. There was extremely significant negative correlation between average temperature and spike length while there was significant positive correlation between average illumination intensity and spike length. The partial correlation coefficient between average temperature and number of effective spikes per plant was bigger $(\mathrm{r}=0.4576)$. Except for duration of day, the correlation between other light-temperature factors and number of effective spikes per plant was significant. The partial correlation coefficient between duration of day and grains per panicle, seed setting rate were bigger which was 0.3746 , -0.5599 , respectively. The partial correlation coefficient between minimum temperature and 1000 grain weight was bigger $(r=-0.1897)$ but the correlation between all the light-temperature factors and 1000 grain weight was not significant.
\end{abstract}

$\underline{\text { Key words: Rice, latitude, light-temperature factors, grain yield, correlation, intensity, China }}$

\section{INTRODUCTION}

At present, food security has become the world issues of common concern. More than half, the population lived on a diet of rice during 1961-2001, the total global rice production, area and yields increased by $171.3,30.9$ and $107.0 \%$. In growth of gross output, contribution of increased harvested area was $27.8 \%$, contribution of increased yield was $72.2 \%$ (Chen and Zhu, 2003). Asia is the major rice production area in term of its total rice production and harvested area, the occupancy of the world was 90 and $91 \%$, respectively. In recent years, $>40$ countries and regions, plant hybrid rice such as Southeast Asia, South Asia, South America, Africa, North America, etc. Hybrid rice has been ranked one of the greatest measures to solve food shortage in developing countries by the FAO, up to the present day, the extension area of hybrid rice added up to 300 million ha in the world which to increase grain production $>500$ million ton. Statistical results showed that the acreage of hybrid rice in Vietnam reached 600,000 ha up to 2003 becoming the 2nd largest rice exporter which was next to Thailand
(Yuan et al., 2008). Because rice is sensitive to light and temperature, the cross-country and cross-regional introduction and cultivation is very difficult. In recent years in order to promote the extension of the hybrid rice, numerous scholars did lots of researches on influences of environmental factors such as temperature, light on the yield and quality of rice. Studies showed that the effects of sunshine from seedling transplanting period to initial heading stage and accumulated temperature from initial heading stage to mature period on the rice yield were significant which main effect the panicle number per hill and seed setting rate (Liu et al., 2009).

The rice yield and yield components were significantly correlated with the accumulated temperature, effective accumulated temperature, extreme temperature difference and duration of day through the growth period of hybrid rice. Light and temperature affected the yield of hybrid rice mainly through their effects on the total and effective numbers of grains spikelet and its fertility. In this aspect, accumulated and effective accumulated temperature played a determinative role during vegetative growth stage while extreme temperature difference and

Corresponding Author: Yang Wen-Yu, Key Laboratory of Crop Culture and Tillage Science of Sichuan Agricultural University, Sichuan, 625014 Ya'an, P.R. China 
duration of day were the key factors during reproductive growth stage (Zhou et al., 2006, 2009). Hiroyuki Shimono studies showed that the higher temperatures, strongger sunlight, higher wind speeds were conducive to the formation of rice yield (Shimono et al., 2007). Shunji Ohta's analysis which based on meteorological and geographical data 2081-2100 showed that between 1.6-2.0 of temperature change will cause rice-growing regions North, planting extended 25-30 days (Ohta and Kimura, 2007). Hak Yoon Kim studies showed that UV-B can significantly affect the rice plant height, tillering, spike number, dry matter accumulation and yield (Kim et al., 1996).

Comprehensive results of previous studies, mostly concentrated in the temperature, UV-B light stress and stress on rice yield. Although, these studies which about the effects of environmental factors on rice quality has made great progress but the material was deficient and few research reports on the effects of accumulated temperature, average illumination intensity and cumulateive illumination intensity factors in each growth developmental stage on rice yield.

For a better understanding of the effects of lighttemperature factors on the grain yield and yield components in different latitude areas, we have done six field experiments in Shanxi Hanzhong, Sichuan Wenjiang, Yunnan Mile of China and Hanoi Wenlin, Zhongbu Guang'ai, Saig on Long'an of Vietnam from 2005-2006. We hope, the results can prefer scientific theoretic for the extension of hybrid paddy rice in the world, especially in the Southeast Asia.

\section{MATERIALS AND METHODS}

Names, parents and sources of the materials are shown in Table 1.

Design of experiment: The experiment was done by six field experiments Shanxi Hanzhong, Sichuan Wenjiang, Yunnan Mile of China and Hanoi Wenlin, Zhongbu Guang'ai, Saigon Long'an of Vietnam from 2005-2006. General situation, entironment and soil nutrition are shown in Table 2. The experiment used randomized completely block design and had two repetitions in each trial area. The block acreage was $10.8 \mathrm{~m}^{2}$, the row spacing was $25 \times 18 \mathrm{~cm}$ with single seedling in each hole. In Shanxi Hanzhong and Sichuan Wenjiang, the sowing time was the last 10 days of April, the transplanting time was the middle 10 days of May, the harvest time was the last 10 days of August in Yunnan Mile, the sowing time was the middle 10 days of May, the transplanting time was the 1 st 10 days of June, the harvest time was the middle 10 days of September.

In Hanoi Wenlin, the sowing time was the 1st 10 days of January, the transplanting time was the 1st 10 days of Febuary, the harvest time was the 1st 10 days of June. In Zhongbu Guang'ai, the sowing time was the last 10 days of December, the transplanting time was the last 10 days of January, the harvest time was the last 10 days of April. In Saigon Long'an, the sowing time was the 1st 10 days of December, the transplanting time was the last 10 days of December, the harvest time was the last 10 days of March. The fertilization, prevention of plant diseases and insect pests were same as local management.

Table 1: Names, parents and sources of materials

\begin{tabular}{|c|c|c|c|}
\hline Codes & Names & Parents & Sources \\
\hline$\overline{A_{1}}$ & Shan you $63(\mathrm{CK})$ & Zhenshan97A/Minghui 63 & Sichuan Jiany ang Yibang Seed Co., Ltd. \\
\hline $\mathrm{A}_{2}$ & Gang you 22 & Gang46A/CDR 22 & Sichuan Agricultural Academy of Sciences \\
\hline $\mathrm{A}_{3}$ & Ji you 1 & G2480A/Shuhui 527 & Sichuan Nongda High-tech. Agriculture Co., Ltd \\
\hline $\mathrm{A}_{4}$ & D you 527 & D62A/Shuhui 527 & Sichuan Nongda High-tech. Agriculture Co., Ltd \\
\hline $\mathrm{A}_{5}$ & Jin you 725 & Jin23A/Mianhui 725 & Sichuan Guohao Seed Co., Ltd. \\
\hline $\mathrm{A}_{6}$ & II you 838 & $-32 \mathrm{~A} /$ Fuhui 838 & Sichuan Jiany ang Yibang Seed Co., Ltd. \\
\hline $\mathrm{A}_{7}$ & D xiang you 26 & DxiangA/6326 & Sichuan Nongda High-tech. Agriculture Co., Ltd \\
\hline $\mathrm{A}_{8}$ & Chuan xiang you 2 & Chuanxiang29A/Chenghui 177 & Sichuan Agricultural Academy of Sciences \\
\hline $\mathrm{A}_{9}$ & Yue you 938 & YuetaiA/R938 & Jiangsu Agricultural Academy of Sciences \\
\hline$A_{10}$ & Xie you 527 & XieqingzhaoA/Shuhui 527 & Sichuan Nongda High-tech. Agriculture Co., Ltd \\
\hline$A_{11}$ & K you 8527 & K18A/Shuhui 527 & Sichuan Agricultural Academy of Sciences \\
\hline $\mathrm{A}_{12}$ & Gang you 527 & Gang46A/Shuhui 527 & Sichuan Nongda High-tech. Agriculture Co., Ltd \\
\hline$\underline{A_{13}}$ & Liang you pei 9 & Peiai64S/9311 & Jiangsu Agricultural Academy of Sciences \\
\hline
\end{tabular}

Table 2: General situation, environment and soil nutrition of each trial area

\begin{tabular}{|c|c|c|c|c|c|c|c|c|}
\hline \multirow[b]{2}{*}{$\underline{\text { Places }}$} & \multirow[b]{2}{*}{$\begin{array}{l}\text { North latitude, East } \\
\text { longitude and altitude (m) }\end{array}$} & \multirow[b]{2}{*}{ Environment } & \multirow[b]{2}{*}{$\begin{array}{l}\text { Humus } \\
(\%)\end{array}$} & \multirow[b]{2}{*}{$\begin{array}{l}\text { Total } \\
\mathrm{N}(\%)\end{array}$} & \multicolumn{4}{|c|}{ Quick ( $\left.\mathrm{mg} \mathrm{kg}^{-1}\right)$} \\
\hline & & & & & $\mathrm{N}$ & $\mathrm{P}$ & $\mathrm{K}$ & $\mathrm{pH}$ \\
\hline Shanxi Hanzhong & $33^{\circ}, 107^{\circ}, 510$ & Northern most of hybrid paddy rice & 3.17 & 0.170 & 143 & 61 & 127 & 6.9 \\
\hline Sichuan Wenjiang & $30.4^{\circ}, 103.5^{\circ}, 520$ & West plain of Sichuan & 3.29 & 0.138 & 188 & 48 & 69 & 5.7 \\
\hline Yunnan Mile & $24.02^{\circ}, 103.2^{\circ}, 1150$ & Semi-tropical with high altitude & 2.49 & 0.153 & 117 & 18 & 73 & 8.0 \\
\hline Hanoi Wenlin & $20.50^{\circ}, 106.6^{\circ}, 5$ & Tropical monsoon climate & 2.50 & 0.140 & 127 & 36 & 55 & 7.0 \\
\hline Zhongbu Guang'ai & $15.09^{\circ}, 108.47^{\circ}, 7$ & Subtropical oceanic climate & 2.75 & 0.122 & 136 & 32 & 60 & 6.8 \\
\hline Saigon Long'an & $10.40^{\circ}, 106.1^{\circ}, 2$ & Tropical maritime climate & 2.60 & 0.137 & 139 & 28 & 65 & 7.5 \\
\hline
\end{tabular}


Grain yield: Take 20 holes to measure number of effective spikes per plant then according to the medium value, take 5 holes to measure panicle length, grain number per panicle, filled grains per panicle, seed setting rate and 1000 grain weight in the end, harvesting the block of rice by manual work, measuring actual grain yield.

Temperature and light: Measured by grapher recording meter (ZDR, produced by Zhejiang University, China)

Statistic analysis: Select Excel 2003, DPS 7.05 as statistical software, multiple comparison method of variance analysis is Duncan analyse.

\section{RESULTS AND DISCUSSION}

Light-temperature factors in rice cropping regions of different latitudes: In rice cropping regions of different latitudes, the maximum temperature was between 24.42 and $31.88^{\circ} \mathrm{C}$, the minimum temperature was between 10.15 and $23.09^{\circ} \mathrm{C}$, the average temperature was between 22.54 and $29.02^{\circ} \mathrm{C}$.

During the whole growing period, the higest effective accumulated temperature was $2286.81^{\circ} \mathrm{C}$, the lowest effective accumulated temperature was $1524.33^{\circ} \mathrm{C}$. Duration of day was between 11.43 and $13.2 \mathrm{~h}$, the average illumination intensity was between 7.39 and 19.36 KLuX; cumulateive illumination intensity was between 983.23 and 2588.67 Klux.

The maximum temperature, minimum temperature, average temperature, effective accumulated temperature, duration of day, average illumination intensity and cumulateive illumination intensity defined as $\mathrm{X}_{1 \cdot 7}$, respectively, grain yield was taken as the induced variable then doing stepwise regression analysis.
Effects of light-temperature factors on the panicle length: Table 3 and 4 shows light-temperature factors as variable while panicle length was the induced variable, multiple stepwise regression analysis showed that there was extremely significant negative correlation between average temperature and spike length while there was significant positive correlation between average illumination intensity and spike length. The function relationship was $\mathrm{Y}=38.7286+0.2551 \mathrm{X}_{1}-0.0423 \mathrm{X}_{2}-0.5409$ $\mathrm{X}_{3}-0.0027 \mathrm{X}_{4}-0.3493 \mathrm{X}_{5}+0.1708 \mathrm{X}_{6}, \mathrm{r}=0.4528>\mathrm{r}_{0.01}=$ $0.2887(\mathrm{n}=77)$.

The size order of partial correlation coefficient between light-temperature factors and panicle length was: average temperature $\left[\mathrm{r}\left(\mathrm{Y}, \mathrm{X}_{3}\right)=-0.2493\right]>$ average illumination intensity $\left[\mathrm{r}\left(\mathrm{Y}, \mathrm{X}_{6}\right)=0.1775\right]>$ effective accumulated temperature $\left[\mathrm{r}\left(\mathrm{Y}, \mathrm{X}_{4}\right)=-0.1213\right]>$ maximum temperature $\left[\mathrm{r}\left(\mathrm{Y}, \mathrm{X}_{1}\right)=0.1051\right]$. The size order of standard regression coefficient was: average temperature $>$ effective accumulated temperature $>$ average illumination intensity $>$ duration of day $>$ maximum temperature $>$ minimum temperature.

Effects of light-temperature factors on grain yield: Table 5 and 6 shows light-temperature factors as variable while grain yield was the induced variable, multiple stepwise regression analysis showed that there was extremely significant positive correlation between four light-temperature factors (effective accumulated temperature, duration of day, average illumination intensity, cumulateive illumination intensity) and grain yield. The function relationship was $\mathrm{Y}=34729.9986$ $-319.6538 \mathrm{X}_{1}-10.470 \mathrm{X}_{2}-384.6741 \mathrm{X}_{3}+1.1804 \mathrm{X}_{4}-1279.8403$ $\mathrm{X}_{5}+160.4714 \mathrm{X}_{6}+2.2498 \mathrm{X}_{7}, \mathrm{r}=0.8435>\mathrm{r}_{0.01}=0.2887(\mathrm{n}=77)$.

Table 3: The related coefficient of panicle length

\begin{tabular}{|c|c|c|c|c|c|c|c|c|c|}
\hline Related coefficient & $\mathrm{X}_{1}$ & $\mathrm{X}_{2}$ & $\mathrm{X}_{3}$ & $\mathrm{X}_{4}$ & $\mathrm{X}_{5}$ & $\mathrm{X}_{6}$ & $\mathrm{X}_{7}$ & $\mathrm{Y}$ & Level of significance $p$ \\
\hline $\mathrm{X}_{1}$ & 1 & & & & & & & & 0.1356 \\
\hline $\mathrm{X}_{2}$ & -0.4784 & 1 & & & & & & & 0.0798 \\
\hline $\mathrm{X}_{3}$ & -0.5830 & 0.6959 & 1 & & & & & & 0.0021 \\
\hline $\mathrm{X}_{4}$ & 0.4465 & -0.3531 & -0.1039 & 1 & & & & & 0.1127 \\
\hline $\mathrm{X}_{5}$ & 0.4058 & -0.4514 & -0.2063 & 0.9227 & 1 & & & & 0.1624 \\
\hline $\mathrm{X}_{6}$ & -0.1622 & 0.2668 & 0.5884 & 0.5680 & 0.4216 & 1 & & & 0.0199 \\
\hline $\mathrm{X}_{7}$ & 0.2693 & -0.0445 & 0.1545 & 0.8725 & 0.7471 & 0.8533 & 1 & & 0.1011 \\
\hline Y & 0.1705 & -0.1996 & $-0.3425^{* * * *}$ & -0.1810 & -0.1597 & $-0.2633^{*}$ & -0.187 & 1 & 0.0001 \\
\hline
\end{tabular}

***Etremely significant, "significant, all the tables are the same meaning

Table 4: The standard regression coefficient of panicle length

\begin{tabular}{llllllrr}
\hline Factor & Immediate & $\rightarrow \mathrm{X}_{1}$ & $\rightarrow \mathrm{X}_{2}$ & $\rightarrow \mathrm{X}_{3}$ & $\rightarrow \mathrm{X}_{4}$ & $\rightarrow \mathrm{X}_{5}$ & $\rightarrow \mathrm{X}_{6}$ \\
\hline $\mathrm{X}_{1}$ & 0.1403 & - & 0.0519 & 0.2630 & -0.1730 & -0.0576 & -0.0541 \\
$\mathrm{X}_{2}$ & -0.1085 & -0.0671 & - & -0.3139 & 0.1368 & 0.0640 & 0.0891 \\
$\mathrm{X}_{3}$ & -0.4511 & -0.0818 & -0.0755 & - & 0.0403 & 0.0293 & 0.1964 \\
$\mathrm{X}_{4}$ & -0.3875 & 0.0626 & 0.0383 & 0.0469 & - & -0.1309 & 0.1896 \\
$\mathrm{X}_{5}$ & -0.1419 & 0.0569 & 0.0490 & 0.0931 & -0.3576 & - & 0.1407 \\
$\mathrm{X}_{3}$ & 0.3338 & -0.0228 & -0.0290 & -0.2654 & -0.2201 & -0.0598 & - \\
\hline
\end{tabular}


Res. J. Agron., 4 (4): 70-77, 2010

Table 5: The related coefficient of grain yield

\begin{tabular}{|c|c|c|c|c|c|c|c|c|c|}
\hline Related coefficient & $x_{1}$ & $x_{2}$ & $\mathrm{X}_{3}$ & $\mathrm{X}_{4}$ & $X_{5}$ & $\mathrm{X}_{6}$ & $\mathrm{X}_{3}$ & $\mathrm{Y}$ & Level of significance $p$ \\
\hline $\mathrm{X}_{1}$ & 1 & & & & & & & & 0.7543 \\
\hline $\mathrm{X}_{2}$ & -0.4784 & 1 & & & & & & & 0.5390 \\
\hline $\mathrm{X}_{3}$ & -0.5830 & 0.6959 & 1 & & & & & & 0.1318 \\
\hline $\mathrm{X}_{4}$ & 0.4465 & -0.3531 & -0.1039 & 1 & & & & & 0.0001 \\
\hline $\mathrm{X}_{5}$ & 0.4058 & -0.4514 & -0.2063 & 0.9227 & 1 & & & & 0.0011 \\
\hline $\mathrm{X}_{6}$ & -0.1622 & 0.2668 & 0.5884 & 0.5680 & 0.4216 & 1 & & & 0.0001 \\
\hline $\mathrm{X}_{7}$ & 0.2693 & -0.0445 & 0.1545 & 0.8725 & 0.7471 & 0.8533 & 1 & & 0.0001 \\
\hline$\underline{\mathrm{Y}}$ & 0.0360 & 0.0706 & 0.1721 & $0.5325^{* * *}$ & $0.3620^{* * *}$ & $0.7391^{* * *}$ & $0.7544^{* * *}$ & 1 & 0.0001 \\
\hline
\end{tabular}

Table 6: The standard regression coefficient of grain yield

\begin{tabular}{|c|c|c|c|c|c|c|c|c|}
\hline Factors & Immediate & $\rightarrow X_{1}$ & $\rightarrow \mathrm{X}_{2}$ & $\rightarrow \mathrm{X}_{3}$ & $\rightarrow \mathrm{X}_{4}$ & $\rightarrow \mathrm{X}_{5}$ & $\rightarrow \mathrm{X}_{6}$ & $\rightarrow \mathrm{X}_{7}$ \\
\hline $\mathrm{X}_{1}$ & -0.2048 & - & 0.015 & 0.2180 & 0.0881 & -0.2458 & -0.0592 & 0.2248 \\
\hline $\mathrm{X}_{2}$ & -0.0313 & 0.098 & - & -0.2602 & -0.0697 & 0.2734 & 0.0975 & -0.0371 \\
\hline $\mathrm{X}_{3}$ & -0.3739 & 0.1194 & -0.0218 & - & -0.0205 & 0.1250 & 0.2149 & 0.1290 \\
\hline $\mathrm{X}_{4}$ & 0.1974 & -0.0915 & 0.0111 & 0.0389 & - & -0.5590 & 0.2075 & 0.7283 \\
\hline $\mathrm{X}_{5}$ & -0.6058 & -0.0831 & 0.0141 & 0.0771 & 0.1821 & - & 0.154 & 0.6235 \\
\hline $\mathrm{X}_{6}$ & 0.3653 & 0.0332 & -0.0084 & -0.2200 & 0.1121 & -0.2554 & - & 0.7122 \\
\hline$x_{7}$ & 0.8346 & -0.0552 & 0.0014 & -0.0578 & 0.1722 & -0.4526 & 0.3117 & \\
\hline
\end{tabular}

Table 7: The related coefficient of effective spikes number per plant

\begin{tabular}{|c|c|c|c|c|c|c|c|c|c|}
\hline Related coefficient & $\mathrm{X}_{1}$ & $\mathrm{X}_{2}$ & $\mathrm{X}_{3}$ & $\mathrm{X}_{4}$ & $\mathrm{X}_{5}$ & $\mathrm{X}_{6}$ & $\mathrm{X}_{7}$ & $\mathrm{Y}$ & Level of significance $p$ \\
\hline $\mathrm{X}_{1}$ & 1 & & & & & & & & 0.0398 \\
\hline $\mathrm{X}_{2}$ & -0.4784 & & & & & & & & 10.0001 \\
\hline $\mathrm{X}_{3}$ & -0.5830 & 0.6959 & 1 & & & & & & 0.0001 \\
\hline $\mathrm{X}_{4}$ & 0.4465 & -0.3531 & -0.1039 & 1 & & & & & 0.0676 \\
\hline $\mathrm{X}_{5}$ & 0.4058 & -0.4514 & -0.2063 & 0.9227 & 1 & & & & 0.2949 \\
\hline $\mathrm{X}_{6}$ & -0.1622 & 0.2668 & 0.5884 & 0.568 & 0.4216 & 1 & & & 0.0001 \\
\hline $\mathrm{X}_{7}$ & 0.2693 & -0.0445 & 0.1545 & 0.8725 & 0.7471 & 0.8533 & 1 & & 0.0001 \\
\hline $\mathrm{Y}$ & $-0.2334 *$ & $0.4807^{* * * *}$ & $0.6354^{* * *}$ & 0.208 & 0.1201 & $0.6818^{* * * *}$ & $0.4934^{* * * *}$ & 1 & 0.0001 \\
\hline
\end{tabular}

Table 8: The standard regression coefficient of effective spikes number per plant

\begin{tabular}{|c|c|c|c|c|c|c|c|c|}
\hline Factors & Immediate & $\rightarrow X_{1}$ & $\rightarrow X_{2}$ & $\rightarrow X_{3}$ & $\rightarrow X_{4}$ & $\rightarrow X_{S}$ & $\rightarrow X_{6}$ & $\rightarrow X_{7}$ \\
\hline $\mathrm{X}_{1}$ & -0.2986 & - & 0.1681 & -0.7959 & -0.6869 & 0.0153 & 0.3795 & 0.9853 \\
\hline $\mathrm{X}_{2}$ & -0.3513 & 0.1429 & - & 0.9499 & 0.5432 & -0.0170 & -0.6243 & -0.1627 \\
\hline $\mathrm{X}_{3}$ & 1.3651 & 0.1741 & -0.2444 & - & 0.1599 & -0.0078 & -1.3766 & 0.5652 \\
\hline $\mathrm{X}_{4}$ & -1.5384 & -0.1333 & 0.1240 & -0.1418 & - & 0.0347 & -1.3289 & 3.1918 \\
\hline $\mathrm{X}_{5}$ & 0.0376 & -0.1212 & 0.1585 & -0.2817 & -1.4196 & - & -0.9864 & 2.7328 \\
\hline $\mathrm{X}_{6}$ & -2.3396 & 0.0484 & -0.0937 & 0.8032 & -0.8738 & 0.0159 & - & 3.1214 \\
\hline $\mathrm{X}_{7}$ & 3.6580 & -0.0804 & 0.0156 & 0.2109 & -1.3424 & 0.0281 & -1.9964 & - \\
\hline
\end{tabular}

Determination coefficient of regression equation between seven light-temperature factors and grain yield was 0.7115 which indicated the linear fitting was befittingly. About $71.5 \%$ variability of grain yield could be caused by the seven light-temperature factors.

The size order of partial correlation coefficient between light-temperature factors and grain yield was: duration of day $\left[\mathrm{r}\left(\mathrm{Y}, \mathrm{X}_{5}\right)=-0.3442\right]>$ maximum temperature $\left[\mathrm{r}\left(\mathrm{Y}, \mathrm{X}_{1}\right)=-0.1682\right]>$ average temperature $\left[\mathrm{r}\left(\mathrm{Y}, \mathrm{X}_{3}\right)=\right.$ $-0.1507]>$ cumulateive illumination intensity $\left[\mathrm{r}\left(\mathrm{Y}, \mathrm{X}_{7}\right)=\right.$ $0.1144]$, the partial correlation coefficient of minimum temperature was the lowest $\left[\mathrm{r}\left(\mathrm{Y}, \mathrm{X}_{2}\right)=-0.0217\right]$.

There was real linear regression between seven light-temperature factors and grain yield. The size order of standard regression coefficient was: cumulateive illumination intensity>duration of day $>$ average temperature $>$ average illumination intensity $>$ maximum temperature $>$ effective accumulated temperature $>$ minimum temperature.
Effects of light-temperature factors on grain yield components

Number of effective spikes per plant: Table 7 and 8 shows light-temperature factors as variable while number of effective spikes per plant was the induced variable, multiple stepwise regression analysis showed that there was extremely significant positive correlation between four light-temperature factors (minimum temperature, average temperature, average illumination intensity) and number of effective spikes per plant. There was significant positive correlation between maximum temperature and number of effective spikes per plant. The function relationship was $\mathrm{Y}=1.0767-0.8797 \mathrm{X}_{1}-0.2218 \mathrm{X}_{2}+2.6509$ $\begin{array}{llllllll}\mathrm{X}_{3} & -0.0174 & \mathrm{X}_{4}+0.1499 & \mathrm{X}_{5} & -1.9401 & \mathrm{X}_{6}+0.0186 & \mathrm{X}_{7}\end{array}$ $\mathrm{r}=0.8139>\mathrm{r}_{0.01}=0.2887(\mathrm{n}=77)$.

There was real linear regression between seven lighttemperature factors and number of effective spikes per plant. The size order of partial correlation coefficient between light-temperature factors and number of effective spikes per plant was; average temperature $\left[\mathrm{r}\left(\mathrm{Y}, \mathrm{X}_{3}\right)=\right.$ 
Res. J. Agron., 4 (4): 70-77, 2010

Table 9: The related coefficient of grain number per panicle

\begin{tabular}{|c|c|c|c|c|c|c|c|c|c|}
\hline Related coefficient & $x_{1}$ & $\mathrm{X}_{2}$ & $\mathrm{X}_{3}$ & $\mathrm{X}_{4}$ & $\mathrm{X}_{\mathrm{S}}$ & $\mathrm{X}_{6}$ & $X_{3}$ & $\mathrm{Y}$ & Level of significance $p$ \\
\hline $\mathrm{X}_{1}$ & 1 & & & & & & & & 0.1250 \\
\hline $\mathrm{X}_{2}$ & -0.4784 & 1 & & & & & & & 0.0389 \\
\hline $\mathrm{X}_{3}$ & -0.583 & 0.6959 & 1 & & & & & & 0.4284 \\
\hline $\mathrm{X}_{4}$ & 0.4465 & -0.3531 & -0.1039 & 1 & & & & & 0.0570 \\
\hline $\mathrm{X}_{5}$ & 0.4058 & -0.4514 & -0.2063 & 0.9227 & 1 & & & & 0.2091 \\
\hline $\mathrm{X}_{6}$ & -0.1622 & 0.2668 & 0.5884 & 0.568 & 0.4216 & 1 & & & 0.2840 \\
\hline $\mathrm{X}_{7}$ & 0.2693 & -0.0445 & 0.1545 & 0.8725 & 0.7471 & 0.8533 & 1 & & 0.1503 \\
\hline$\underline{Y}$ & 0.1752 & $-0.2344^{*}$ & -0.0910 & 0.2165 & 0.1438 & 0.1228 & 0.1644 & 1 & 0.0001 \\
\hline
\end{tabular}

Table 10: The standard regression coefficient of grain number per panicle

\begin{tabular}{llllllrrr}
\hline Factors & Immediate & $\rightarrow \mathrm{X}_{1}$ & $\rightarrow \mathrm{X}_{2}$ & $\rightarrow \mathrm{X}_{3}$ & $\rightarrow \mathrm{X}_{4}$ & $\rightarrow \mathrm{X}_{5}$ & $\rightarrow \mathrm{X}_{6}$ & $\rightarrow \mathrm{X}_{7}$ \\
\hline $\mathrm{X}_{1}$ & 0.6262 & - & -0.1940 & 0.8082 & 0.8691 & -0.0786 & -0.6200 & -1.2357 \\
$\mathrm{X}_{2}$ & 0.4055 & -0.2996 & - & -0.9646 & -0.6873 & 0.0874 & 1.0200 & 0.2041 \\
$\mathrm{X}_{3}$ & -1.3861 & -0.3651 & 0.2821 & - & -0.2023 & 0.0400 & 2.2493 & -0.7088 \\
$\mathrm{X}_{4}$ & 1.9465 & 0.2796 & -0.1432 & 0.1440 & - & -0.1788 & 2.1713 & -4.0031 \\
$\mathrm{X}_{5}$ & -0.1937 & 0.2541 & -0.1830 & 0.2860 & 1.7961 & - & -0.017 & -3.4274 \\
$\mathrm{X}_{6}$ & 3.8228 & -0.1016 & 0.1082 & -0.8156 & 1.1056 & -0.0817 & - & -3.9149 \\
$\mathrm{X}_{3}$ & -4.5878 & 0.1687 & -0.0180 & -0.2142 & 1.6984 & -0.1447 & 3.2620 & - \\
\hline
\end{tabular}

Table 11: The related coefficient of seed setting rate

\begin{tabular}{|c|c|c|c|c|c|c|c|c|c|}
\hline Related coefficient & $x_{1}$ & $\mathrm{X}_{2}$ & $\mathrm{x}_{3}$ & $\mathrm{X}_{4}$ & $\mathrm{X}_{5}$ & $\mathrm{X}_{6}$ & $\mathrm{X}_{7}$ & $\mathrm{Y}$ & Level of significance $p$ \\
\hline $\mathrm{X}_{1}$ & 1 & & & & & & & & 0.1674 \\
\hline $\mathrm{X}_{2}$ & -0.4784 & 1 & & & & & & & 0.8727 \\
\hline $\mathrm{X}_{3}$ & -0.5830 & 0.6959 & 1 & & & & & & 0.0552 \\
\hline $\mathrm{X}_{4}$ & 0.4465 & -0.3531 & -0.1039 & 1 & & & & & 0.1196 \\
\hline $\mathrm{X}_{5}$ & 0.4058 & -0.4514 & -0.2063 & 0.9227 & 1 & & & & 0.0019 \\
\hline $\mathrm{X}_{6}$ & -0.1622 & 0.2668 & 0.5884 & 0.5680 & 0.4216 & 1 & & & 0.8984 \\
\hline $\mathrm{X}_{7}$ & 0.2693 & -0.0445 & 0.1545 & 0.8725 & 0.7471 & 0.8533 & 1 & & 0.9183 \\
\hline$\underline{Y}$ & 0.1579 & -0.0184 & -0.2180 & -0.1777 & $-0.3458^{* *}$ & -0.0147 & -0.0118 & 1 & 0.0001 \\
\hline
\end{tabular}

$0.4576]>$ effective accumulated temperature $\left[\mathrm{r}\left(\mathrm{Y}, \mathrm{X}_{4}\right)=\right.$ $-0.4251]>$ cumulateive illumination intensity $\left[\mathrm{r}\left(\mathrm{Y}, \mathrm{X}_{7}\right)=\right.$ $0.4229]$ average illumination intensity $\left[\mathrm{r}\left(\mathrm{Y}, \mathrm{X}_{6}\right)=-0.346\right]$. According to the standard regression coefficient, the size order was cumulateive illumination intensity>average illumination intensity $>$ effective accumulated temperature $>$ average temperature $>$ minimum temperature $>$ maximum temperature $>$ duration of day.

Grain number per panicle: Table 9 and 10 shows lighttemperature factors as variable while grain number per panicle was the induced variable, multiple stepwise regression analysis showed that there was significant positive correlation between minimum temperature and grain number per panicle. The function relationship was $\mathrm{Y}=5.7505+11.2535 \mathrm{X}_{1}+1.5616 \mathrm{X}_{2}-16.4214 \mathrm{X}_{3}+0.1341 \mathrm{X}_{4}$ $-4.7128 \mathrm{X}_{5}+19.3379 \mathrm{X}_{6}-0.1424 \mathrm{X}_{7}, \mathrm{r}=0.4994>\mathrm{r}_{0.01}=0.2887$ $(\mathrm{n}=77)$.

The size order of partial correlation coefficient between light-temperature factors and grain number per panicle was: average illumination intensity $\left[\mathrm{r}\left(\mathrm{Y}, \mathrm{X}_{6}\right)=\right.$ $0.3746]>$ effective accumulated temperature $\left[\mathrm{r}\left(\mathrm{Y}, \mathrm{X}_{4}\right)=\right.$ $0.3702]>$ cumulateive illumination intensity $\left[\mathrm{r}\left(\mathrm{Y}, \mathrm{X}_{7}\right)=\right.$ $-0.3654]>$ average temperature $\left[\mathrm{r}\left(\mathrm{Y}, \mathrm{X}_{3}\right)=-0.3307\right]$ $>$ maximum temperature $\left[\mathrm{r}\left(\mathrm{Y}, \mathrm{X}_{1}\right)=0.3078\right]$. According to the standard regression coefficient, the size order was:
Table 12: The standard regression coefficient of seed setting rate

\begin{tabular}{llllllll}
\hline Factors & Immediate & $\rightarrow \mathrm{X}_{1}$ & $\rightarrow \mathrm{X}_{2}$ & $\rightarrow \mathrm{X}_{3}$ & $\rightarrow \mathrm{X}_{4}$ & $\rightarrow \mathrm{X}_{5}$ & $\rightarrow \mathrm{X}_{6}$ \\
\hline $\mathrm{X}_{1}$ & 0.1582 & - & 0.0293 & 0.4721 & 0.158 & -0.5225 & -0.1372 \\
$\mathrm{X}_{2}$ & -0.0613 & -0.0757 & - & -0.5635 & -0.1249 & 0.5812 & 0.2257 \\
$\mathrm{X}_{3}$ & -0.8097 & -0.0922 & -0.0426 & - & -0.0368 & 0.2657 & 0.4976 \\
$\mathrm{X}_{4}$ & 0.3538 & 0.0706 & 0.0216 & 0.0841 & - & -1.1882 & 0.4804 \\
$\mathrm{X}_{5}$ & -1.2877 & 0.0642 & 0.0277 & 0.1671 & 0.3264 & - & 0.3566 \\
$\mathrm{X}_{6}$ & 0.8457 & -0.0257 & -0.0163 & -0.4765 & 0.2009 & -0.5429 & - \\
\hline
\end{tabular}

cumulateive illumination intensity $>$ average illumination intensity $>$ effective accumulated temperature $>$ average temperature $>$ maximum temperature $>$ minimum temperature $>$ duration of day.

Seed setting rate: Table 11 and 12 shows lighttemperature factors as variable while seed setting rate was the induced variable, multiple stepwise regression analysis showed that there was extremely significant positive correlation between duration of day and seed setting rate. The function relationship was $\mathrm{Y}=203.4796$ $+0.7321 \mathrm{X}_{1}-0.06078 \mathrm{X}_{2}-2.4712 \mathrm{X}_{3}+0.0062 \mathrm{X}_{4}-8.0697 \mathrm{X}_{5}$ $+1.1021 \mathrm{X}_{6}, \mathrm{r}=0.7567>\mathrm{r}_{0.01}=0.2887(\mathrm{n}=77)$.

The size order of partial correlation coefficient between the selected six light-temperature factors and seed setting rate was: duration of day $\left[\mathrm{r}\left(\mathrm{Y}, \mathrm{X}_{5}\right)=-0.5599\right]$ >average temperature $\left[\mathrm{r}\left(\mathrm{Y}, \mathrm{X}_{3}\right)=-0.5332\right]>$ average illumination intensity $\left[\mathrm{r}\left(\mathrm{Y}, \mathrm{X}_{6}\right)=0.529\right]$. According to the standard regression coefficient, the size order was: 
Res. J. Agron., 4 (4): 70-77, 2010

Table 13: The related coefficient of 1000 -grain weight

\begin{tabular}{|c|c|c|c|c|c|c|c|c|c|}
\hline Related coefficient & $X_{1}$ & $\mathrm{X}_{2}$ & $\mathrm{X}_{3}$ & $\mathrm{X}_{4}$ & $\mathrm{X}_{5}$ & $X_{6}$ & $\mathrm{X}_{7}$ & $\mathrm{Y}$ & Level of significance $p$ \\
\hline $\mathrm{X}_{1}$ & 1 & & & & & & & & 0.4259 \\
\hline $\mathrm{X}_{2}$ & -0.4784 & 1 & & & & & & & 0.1195 \\
\hline $\mathrm{X}_{3}$ & -0.5830 & 0.6959 & 1 & & & & & & 0.1219 \\
\hline $\mathrm{X}_{4}$ & 0.4465 & -0.3531 & -0.1039 & 1 & & & & & 0.5850 \\
\hline $\mathrm{X}_{5}$ & 0.4058 & -0.4514 & -0.2063 & 0.9227 & 1 & & & & 0.5059 \\
\hline $\mathrm{X}_{6}$ & -0.1622 & 0.2668 & 0.5884 & 0.5680 & 0.4216 & 1 & & & 0.9421 \\
\hline $\mathrm{X}_{7}$ & 0.2693 & -0.0445 & 0.1545 & 0.8725 & 0.7471 & 0.8533 & 1 & & 0.4781 \\
\hline$\underline{\mathrm{Y}}$ & 0.0915 & -0.1777 & -0.1766 & 0.0628 & 0.0764 & 0.0084 & 0.0815 & 1 & 0.0001 \\
\hline
\end{tabular}

Table 14: The standard regression coefficient of 1000 -grain weight

\begin{tabular}{|c|c|c|c|c|c|c|c|c|}
\hline Factors & Immediate & $\rightarrow X_{1}$ & $\rightarrow \mathrm{X}_{2}$ & $\rightarrow X_{3}$ & $\rightarrow \mathrm{X}_{4}$ & $\rightarrow X_{5}$ & $\rightarrow \mathrm{X}_{6}$ & $\rightarrow X_{7}$ \\
\hline$X_{1}$ & -0.2817 & - & 0.2362 & -0.2218 & -0.4377 & -0.0562 & 0.2374 & 0.6153 \\
\hline $\mathrm{X}_{2}$ & -0.4938 & 0.1348 & - & 0.2647 & 0.3461 & 0.0626 & -0.3905 & -0.1016 \\
\hline $\mathrm{X}_{3}$ & 0.3804 & 0.1642 & -0.3436 & - & 0.1019 & 0.0286 & -0.8610 & 0.3529 \\
\hline $\mathrm{X}_{4}$ & -0.9803 & -0.1258 & 0.1743 & -0.0395 & - & -0.1279 & -0.8312 & 1.9932 \\
\hline $\mathrm{X}_{5}$ & -0.1386 & -0.1143 & 0.2229 & -0.0785 & -0.9046 & - & -0.6170 & 1.7065 \\
\hline $\mathrm{X}_{6}$ & -1.4634 & 0.0457 & -0.1318 & 0.2238 & -0.5568 & -0.0584 & - & 1.9492 \\
\hline$\underline{X_{3}}$ & 2.2843 & -0.0759 & 0.0220 & 0.0588 & -0.8554 & -0.1036 & -1.2487 & - \\
\hline
\end{tabular}

duration of day>average illumination intensity $>$ average temperature $>$ effective accumulated temperature $>$ maximum temperature $>$ minimum temperature.

1000-grain weight: Table 13 and 14 shows lighttemperature factors as variable while 1000-grain weight was the induced variable, multiple stepwise regression analysis showed that the positive correlation between seven light-temperature factors and seed setting rate was indistinctively. The function relationship was $\mathrm{Y}=53.2899$ $\begin{array}{llll}-0.5706 \mathrm{X}_{1} & -0.2144 \mathrm{X}_{2}+0.5079 \mathrm{X}_{3} & -0.0076 \mathrm{X}_{4} & -0.3802 \mathrm{X}_{5}\end{array}$ $-0.8345 \mathrm{X}_{6}+0.0079 \mathrm{X}_{7}, \mathrm{r}=0.3108>\mathrm{r}_{0.05}=0.2187(\mathrm{n}=77)$.

The size order of partial correlation coefficient between the selected seven light-temperature factors and 1000 -grain weight was: minimum temperature $\left[\mathrm{r}\left(\mathrm{Y}, \mathrm{X}_{2}\right)=\right.$ -0.1897] >effective accumulated temperature $\left[\mathrm{r}\left(\mathrm{Y}, \mathrm{X}_{4}\right)=\right.$ $-0.1799]>$ cumulateive illumination intensity $\left[\mathrm{r}\left(\mathrm{Y}, \mathrm{X}_{7}\right)=\right.$ $0.1754>$ average illumination intensity $\left[r\left(Y, X_{6}\right)=-0.1396\right]$ $>$ maximum temperature $\left[\mathrm{r}\left(\mathrm{Y}, \mathrm{X}_{1}\right)=-0.1315\right]$. According to the standard regression coefficient, the size order was: cumulateive illumination intensity>average illumination intensity $>$ effective accumulated temperature $>$ minimum temperature $>$ average temperature $>$ maximum temperature $>$ duration of day.

For the past many years, in order to increase crop yield, many scientific researchers probe into the factors affecting crop yield by different ways such as correlation analysis, path analysis, regression analysis and grey relational grade, etc. Up to now, most research focus on the crop economic character, namely crop genetics and breeding but less of research focus on the crop growing environment such as illumination, temperature, elevation, etc. (Liu and Wu, 1995; Diao et al., 1999; Bai and Zhang, 2001; Shen et al., 2000). Crop varieties growing in a specific environment, apart from influenced by genetic characteristics, its growth and yield formation were also influenced by environmental factors which often determine the growth and yield performance such as illumination, temperature, elevation, moisture, etc. (Liu et al., 2003; Wang and Man, 2006; Yuan et al., 2008). In fact, different crop varieties have consistent yield performance in different locations, there was significant genotype $\mathrm{x}$ environment interactions. If a variety $\mathrm{x}$ location interaction were great and the role of in the same direction with suitable matching, the variety has higher yield than the other in the location.

By principal component analysis and regression analysis, Ji Biaojun considered that there was a great influence of the latitude on the yield of rice. In this study, the maximum temperature, minimum temperature, average temperature, effective accumulated temperature, duration of day, average illumination intensity and cumulateive illumination intensity defined as $\mathrm{X}_{1-7}$, respectively, grain yield was taken as the induced variable then doing stepwise regression analysis. The results showed as follows: there was real linear regression between seven light-temperature factors and grain yield. The size order of standard regression coefficient was: cumulateive illumination intensity $>$ duration of day $>$ average temperature $>$ average illumination intensity $>$ maximum temperature $>$ effective accumulated temperature $>$ minimum temperature. There was extremely significant negative correlation between average temperature and spike length while there was significant positive correlation between average illumination intensity and spike length. The partial correlation coefficient between average temperature and number of effective spikes per plant was bigger $(r=0.4576)$. Except for duration of day, the correlation between other light-temperature factors and number of effective spikes per plant was significant. The 
partial correlation coefficient between duration of day and grains per panicle, seed setting rate were bigger which was $0.3746,-0.5599$, respectively. The partial correlation coefficient between minimum temperature and 1000-grain weight was bigger $(r=-0.1897)$ but the correlation between all the light-temperature factors and 1000-grain weight was not significant.

Therefore, the research considered that the factor of temperature and light primarily through the influence of the panicles per plant and the seed setting rate on the yield of rice finally in according with the results of Liu Bo, Xie Xiao Wen. It confirm that the conclusion once again. At the same time, the study further shows that the minimum temperature, average temperature, average illumination intensity, cumulateive illumination intensity affected the number of effective spikes per plant significantly, the duration of day significantly affected the seed set rate. Therefore in different rice ecological zone according to their ecological conditions, identified the limiting factors in rice yields, it can be reduced by selecting varieties and cultivation practices. For example in cold areas, it should be mainly to overcome the effect of temperature on the effective panicles per plant use the species which have good ability of tillering, proper close planting to increase the effective ear and then obtain high yields.

In areas of lacking light such as Sichuan, Chongqing, Guizhou, rice growth often encountered few days rain in later, resulting in decreased seed setting rate and rice yields. If using the varieties which has higher seed set rate and appropriate growth season, adopting to increase the $\mathrm{P}$ and $\mathrm{K}$-fertilizer, one can also get higher yields. In this experimental study, the varieties of Dyou 527, Xieyou 527 and Gangyou 527 have good performance in the various ecological zones.

In which, Dyou 527 through the state approved in 2003 , Xieyou 527 through the state approved in 2004, they are selected to become super rice in China and become major high-yield, high-quality varieties in in Southwest of China and Hanoi Vietnam.

Considering the mechanism of the influence of lighttemperature factors on grain yield, Xu Xianbin find that the different accumulated directly affects the dry weight of rice S-curve inflection point, especially in heading date, flowering date and filling date, there was positively correlated between accumulated temperature and source/leaf, negatively correlated between accumulated temperature and the library/year (Xu et al., 2002). The reasones may be, it affect the energy utilization of rice population, causing differences in tillering which the average illumination, illumination of the cumulative affect the number of effective panicles. But in a different light conditions, the leaf structure of rice population and distribution of light still needs further study. The reason on the length of sunshine affecting seed setting rate may be that insufficient light has greatly affected the production and accumulation of nutrients, thus affecting the panicle initiation and development to promote degradation of spikelets, reduce the grain number, increase the rate of shell.

In addition, this study shows that the influence of temperature and light on the rice grain weight were not significant, this may be that grain weight more affected by genetic factors and have less relationship with temperature and light (Xu et al., 2002).

\section{ACKNOWLEDGEMENT}

The researcher guarantee the authenticity of the study. Funding was provided by Science and Technology Engineering Project of China on grain high yield (2006 BAD 02A05).

\section{REFERENCES}

Bai, L.M. and F.M. Zhang, 2001. Grey correlation analysis of yield and yield components in rice. Syst. Sci. Comprehensive Stud. Agric., 17: 65-66.

Chen, H.Z. and D.F. Zhu, 2003. General situation of global rice production and ecological system. Hybrid Rice, 18: 1-4.

Diao, L.P., J.F. Wang, Y.S. Li, H.N. Zhang, H.S. Zhang, H.Q. Zhai and T.N. Yang, 1999. Analysis of main agronomic characters for Japonica rice from Taihu Lake Region. J. Nanjing Agric. Univ., 22: 1-4.

Kim, H.Y., K. Kobayashi, I. Nouchi and T. Yoneyama, 1996. Enhanced UV-B radiation has little effect on growth, ä13C values and pigments of pot-grown rice (Oryza sativa) in the field. Physiol. Plantarum, 96: 1-5.

Liu, B., Y. Han, J.G. Li and Q. Gao, 2009. Effect of environmental factors on quality of rice. North Rice, 39: $10-12$.

Liu, H.B. and W. Wu, 1995. Multivariate statistics analysis of rice determination factor. Res. Soil Water Conser., 2: 51-55.

Liu, J.X., A.G. Guo and H.L. Guo, 2003. Morphological variation and types of Cynodon dactylon. Acta Prataculturae Sinica, 12: 99-104.

Ohta, S. and A. Kimura, 2007. Impacts of climate changes on the temperature of paddy waters and suitable land for rice cultivation in Japan. Agric. Forest Meteorol., 147: 186-198. 
Shen X.H., S.H. Yang, F.X. Xie and L. Wang, 2000. Genotype $\times$ environment interaction and its relationship to climate factors in rice regional trial. Chinese J. Rice Sci., 14: 31-36.

Shimono, H., T. Hasegawa, T. Kuwagata and K. Iwama, 2007. Modeling the effects of water temperature on rice growth and yield under a cool climate: II. model application. Agron. J., 99: 1338-1344.

Wang, Y.G. and A.Q. Man, 2006. Differing response to drought stress of two Cynodon dactylon varieties. Acta Prataculturae Sinica, 15: 58-64.

Xu, J.L., Q.Z. Xue, L.J. Luo and Z.K. Li, 2002. Genetic dissection of grain weight and its related traits in rice (Oryza sativa L.). Chinese J. Rice Sci., 16: 6-10.
Yuan, J.C., S.M. Yang, M.T. Wang, Y.C. Wu, Q.S. Zhu and J.C. Yang, 2008. Changes in growth duration of rice with altitude and its temperature accumulation effect in Panxi region, China. Acta Agronomica Sinica, 34: 247-253.

Zhou, H., J. Guo, H. Li, T. Wu and J. Zhang, 2006. Canonical correlations of light and temperature with yield and quality characters of F1 ecological populations of hybrid rice. Ying Yong Sheng Tai Xue Bao., 17: 663-667.

Zhou, H.K., Z.P. Cao, C.H. Ye, J.M. He, R.F. Guo, J.F. Guo and F.S. Li, 2009. Factor analysis of yield and quality characters of hybrid rice. Chinese J. Trop. Crops, 30: $147-152$ 